\title{
How does susceptibility to HTLV-1 infection varies with the maturation state of dendritic cells?
}

\author{
Gerges Rizkallah¹,2, Chloé Journo1,2, Renaud Mahieux ${ }^{1,2}$ \& Hélène Dutartre*,1,2 \\ ${ }^{1}$ International Center for Research in Infectiology, Retroviral Oncogenesis laboratory, INSERM U1111 - Université Claude Bernard \\ Lyon 1, CNRS, UMR5308, Ecole Normale Supérieure de Lyon, Université Lyon, F-69007, Lyon, France \\ 2Equipe labellisée "Ligue Nationale Contre le Cancer" \\ * Author for correspondence: helene.dutartre@ens-lyon.fr
}

\section{'Taken together, our results suggest that restriction of HTLV-1 infection in mature DCs occurs after viral capture but before viral integration"}

First draft submitted: 12 June 2017; Accepted for publication: 3 July 2017; Published online: 11 October 2017

Keywords: dendritic cells $\bullet$ dendritic cell maturation $\bullet$ HTLV-1 infection $\bullet$ viral infection

Human T-lymphotropic virus type 1 (HTLV-1) is the etiological agent of adult T-cell leukemia/lymphoma and HTLV-1-associated myelopathy. In patients, the provirus is detected in CD4 ${ }^{+} \mathrm{T}$ cells. HTLV-1 also infects blood or monocyte-derived dendritic cells (DCs) in vitro. Since DCs are more susceptible to HTLV-1 infection than autologous $T$ cells, it has been suggested that they might be intermediaries for the viral spread to lymphocytes. DCs can switch from an immature to a mature state after contact with pathogens. In other viral infection, mature DCs are more efficient than immature DCs in viral transmission to T cells. In this editorial, we highlight a work demonstrating that mature DCs are unable to transmit HTLV-1 to T cells and discuss the mechanism of such restriction.

HTLV-1 was identified as the first human oncogenic retrovirus [1,2]. It is the etiological agent of adult T-cell leukemia (ATL), an oligoclonal malignancy of infected $\mathrm{CD}^{+} \mathrm{T}$ lymphocytes, and of tropical spastic paraparesis, a chronic progressive inflammatory disease. Five to ten million individuals are HTLV-1 carriers worldwide [3], a probably underestimated number. HTLV-1 inter-individual transmission relies on mother-to-child transmission through prolonged breastfeeding, on sexual transmission and on transmission with contaminated blood products [4].

In chronically infected individuals, HTLV-1 proviral DNA is mainly found in $\mathrm{CD}^{+} \mathrm{T}$ cells, but several reports have demonstrated that others immune cell types are infected by HTLV-1 in vivo [5-7]. These cell types include DCs, which are specialized antigen-presenting cells and essential mediators in shaping innate and adaptive immunity [8]. In vitro studies conducted by our lab and others confirmed that HTLV-1 productively infects myeloid blood DCs [7] and monocyte-derived DCs (MDDCs) [9-11]. Of note, exposure of target cells to cell-free viruses only leads to very low levels of productive infection [9], consistent with the observation that efficient infection of target cells requires close cell-to-cell contacts with infected donor cells [12]. Viral entry in T cells requires expression of the HTLV-1 receptor complex, in other words, neuropilin-1 and heparan sulfate proteoglycans, used for binding [13], and the glucose transporter Glut-1, required for fusion [14], while viral binding to DCs was also shown to require DC-SIGN [11].

Importantly, we demonstrated that primary MDDCs are more susceptible to productive HTLV-1 infection than their autologous $T$ cells [9]. Given that productively infected MDDCs are able to transfer HTLV-1 to T cells [7,9,11], it is thus proposed that DCs might be important intermediaries for HTLV-1 spread to T cells in newly infected individuals.

Upon stimulation, DCs can switch from an immature to a mature state characterized by morphological, phenotypic and functional changes [15]. Interestingly, in the context of HIV-1, another retrovirus that spreads from DCs to T cells in newly infected individuals, mature DCs have been shown to be more efficient than immature DCs in viral transmission to T cells [16]. Since interaction of HTLV-1 with mature DCs has not been described 
yet, we examined in a recent work whether immature or mature MDDCs have the same susceptibility to HTLV-1 infection and replication and whether they have the same ability to transfer HTLV-1 to T cells [17].

Immature MDDCs were differentiated from primary monocytes in the presence of IL- 4 and GM-CSF. Maturation was induced either by replacing IL- 4 by IFN- $\alpha$ in the differentiation medium (IFN- $\alpha$ DCs), or by stimulating immature IL-4 DCs with lipopolysaccharide (LPS), a toll-like receptor 4 agonist [18]. While immature DCs were productively infected by HTLV-1, LPS-matured DCs and IFN- $\alpha$ DCs were three- and sixfold less susceptible, respectively. More importantly, although HTLV-1 capture was higher in mature DCs compared with immature DCs, viral transfer to $T$ cells was much reduced in these subsets. Thus, in contrast to immature DCs, mature DCs subsets capture HTLV-1 efficiently, but then restrict both HTLV-1 productive infection and HTLV-1 transmission to $T$ cells.

To further characterize how DC maturation could affect susceptibility to HTLV-1 infection, immature DCs were treated with different toll-like receptor agonists, known to induce distinct maturation phenotypes [18]. This led to the observation that the strength of HTLV-1 restriction correlates the level of DC maturation. Because mature DCs produce high amounts of IFN- $\alpha$ and because it is well established that IFN- $\alpha$ has anti-HTLV-1 activity in other cell types $[19,20]$, we then asked whether HTLV-1 restriction in mature DCs was due to the antiviral effect of IFN- $\alpha$. Surprisingly, neither treatment of immature DCs with recombinant IFN- $\alpha$ nor culture with supernatant from mature DCs (that contains high amounts of physiological type I IFNs) was able to restrict HTLV-1 infection. This is in contrast with previous studies $[19,20]$ and suggests that therapeutic failure of type I IFN therapy used in some ATL chemotherapy protocols [21] could be due to resistance of DCs to IFN- $\alpha$ treatment. This hypothesis would need confirmation through clinical trials monitoring the proviral load in DCs from ATL patients throughout anti-viral therapy.

To decipher the mechanism of HTLV-1 restriction in mature DCs, we analyzed the localization of captured HTLV-1 particles in immature or mature DCs. In all DC subsets, confocal imaging localized HTLV-1 in vesicles positive for CD82, a tetraspanin marker located in multivesicular bodies, but negative for early endosome antigen marker (EEA1). Of note, CD82 is known to localize at the plasma membrane and to interact with HTLV-1 envelope SU glycoprotein in T cells [22]. Thus, in addition to its interaction with HTLV-1 receptor complex containing Glut-1 [23], HTLV-1 Env interaction with CD82 could direct HTLV-1 to unconventional compartments. However, because HTLV-1 localization is similar in immature and mature DCs, targeting of incoming particles to specific compartments cannot explain viral restriction in mature DCs. We next hypothesized that different trafficking routes might deliver HTLV-1 to vesicles with similar markers, yet with distinct physical properties. We determined viral trafficking pathways in the different DCs subsets using pharmacological inhibitors that block clathrin-mediated endocytosis, caveolin-mediated endocytosis or macropinocytosis. These pathways are used by several viruses as main entry pathways in DCs [24]. In immature DCs, HTLV-1 entry followed both clathrin-mediated endocytosis and macropinocytosis, while in IFN- $\alpha$ DCs, HTLV-1 entry followed only clathrin-mediated endocytosis. Moreover, although HTLV-1 entry in LPS-matured DCs used macropinocytosis as in immature DCs, HTLV-1 capture decreased more strongly after treatment with tyrosine kinase receptor inhibitors, PKC inhibitor or Rac 1 inhibitors in LPS-matured DCs than in immature DCs. These results suggest that HTLV-1 entry in mature DCs might follow a different trafficking route and that this could impact the infection outcome.

Low $\mathrm{pH}$ of endocytosis vesicles is characteristic of mature DCs [25]. Using pharmacological agents that modify $\mathrm{pH}$ of vesicles, we showed that productive infection of immature DCs is abolished upon vesicle acidification. In contrast, vesicles neutralization in matured DCs (both LPS-treated DCs and IFN- $\alpha$ DCs) partially restored their productive infection as well as their ability to transmit HTLV-1 to T cells. This shows that the $\mathrm{pH}$ of the compartments in which HTLV-1 is stored after capture determines the efficiency of productive infection. This could be due to instability of HTLV-1 envelope under acidic conditions, thus impairing the subsequent Glut-1dependent fusion with the cellular membrane and viral capsid release in the cell cytoplasm [23]. This would explain the absence of proviral DNA detection in mature DCs [10].

Taken together, our results suggest that restriction of HTLV-1 infection in mature DCs occurs after viral capture but before viral integration. Because neutralization of vesicles in mature DCs only partially restores productive infection, it can be hypothesized that complete restriction might also rely on the expression of some restriction factors, or on the lack of expression of cellular factors required for infection, in mature DCs. Indeed, it has been shown that different DC subsets or differently activated DCs have distinct transcriptomic programs [18]. One candidate could be the binding protein DC-SIGN. Interestingly, we observed that its expression is high in immature DCs but decreases in LPS-matured DCs and IFN- $\alpha$ DCs. Furthermore, signaling through DC-SIGN is required 
for HIV-1 productive infection in DCs [26]. Thus, interaction with and/or signaling through DC-SIGN could be required not only for HTLV-1 entry [11], but also for productive infection of immature DCs. If so, DC-SIGN blockade could constitute a potential targeted therapy for limiting the productive infection of immature DCs and also the subsequent viral transmission to T cells.

In conclusion, our work is the first to our knowledge to describe differences in susceptibility among DC subtypes to HTLV-1 infection, thus providing a better understanding of HTLV-1 interactions with DCs. Because such interactions might be crucial for the in vivo spread of HTLV-1 and for the progression from asymptomatic carriage towards development of HTLV-1-related diseases, targeting infection of DCs could offer new therapeutic approaches.

\section{Financial \& competing interests disclosure}

This work was supported by the "Ligue Nationale contre le Cancer, équipe labélisée program" to R Mahieux. G Rizkallah is funded by a PhD grant from a joint funding program from the Lebanese National Council for Scientific Research CNRS-L and the Université Saint-Joseph USJ and from Fondation Merieux. H Dutartre is funded by INSERM, C Journo and R Mahieux are funded by the ENS de Lyon. The funders had no role in study design, data collection and analysis, decision to publish, or preparation of the manuscript. The authors have no other relevant affiliations or financial involvement with any organization or entity with a financial interest in or financial conflict with the subject matter or materials discussed in the manuscript apart from those disclosed.

No writing assistance was utilized in the production of this manuscript.

\section{References}

1 Poiesz BJ, Ruscetti FW, Gazdar AF, Bunn PA, Minna JD, Gallo RC. Detection and isolation of type C retrovirus particles from fresh and cultured lymphocytes of a patient with cutaneous T-cell lymphoma. Proc. Natl Acad. Sci. USA 77(12), 7415-7419 (1980).

2 Yoshida M, Miyoshi I, Hinuma Y. Isolation and characterization of retrovirus from cell lines of human adult T-cell leukemia and its implication in the disease. Proc. Natl Acad. Sci. USA 79(6), 2031-2035 (1982).

3 Gessain A, Cassar O. Epidemiological aspects and world distribution of HTLV-1 infection. Front. Microbiol. 3 , 388 (2012).

4 Pique C, Jones KS. Pathways of cell-cell transmission of HTLV-1. Front. Microbiol. 3, 378 (2012).

5 Nascimento CR, Lima MA, de Andrada Serpa MJ, Espindola O, Leite ACC, Echevarria-Lima J. Monocytes from HTLV-1-infected patients are unable to fully mature into dendritic cells. Blood 117(2), 489-499 (2011).

6 Koyanagi Y, Itoyama Y, Nakamura N et al. In vivo infection of human T-cell leukemia virus type I in non-T cells. Virology 196(1), 25-33 (1993).

7 Jones KS, Petrow-Sadowski C, Huang YK, Bertolette DC, Ruscetti FW. Cell-free HTLV-1 infects dendritic cells leading to transmission and transformation of CD4 ${ }^{(+)}$T cells. Nat. Med. 14(4), 429-436 (2008).

8 Steinman RM, Hemmi H. Dendritic cells: translating innate to adaptive immunity. Curr. Top. Microbiol. Immunol. 311, 17-58 (2006).

9 Alais S, Mahieux R, Dutartre H. Viral source-independent high susceptibility of dendritic cells to human T-cell Leukemia virus type 1 infection compared to that of T lymphocytes. J. Virol. 89(20), 10580-10590 (2015).

10 Ceccaldi P-E, Delebecque F, Prevost M-C et al. DC-SIGN facilitates fusion of dendritic cells with human T-cell leukemia virus type 1-infected cells. J. Virol. 80(10), 4771-4780 (2006).

11 Jain P, Manuel SL, Khan ZK, Ahuja J, Quann K, Wigdahl B. DC-SIGN mediates cell-free infection and transmission of human T-cell lymphotropic virus type 1 by dendritic cells. J. Virol. 83(21), 10908-10921 (2009).

12 Igakura T, Stinchcombe JC, Goon PKC et al. Spread of HTLV-I between lymphocytes by virus-induced polarization of the cytoskeleton. Science 299(5613), 1713-1716 (2003).

13 Ghez D, Lepelletier Y, Lambert S et al. Neuropilin-1 is involved in human T-cell lymphotropic virus type 1 entry. J. Virol. 80(14), 6844-6854 (2006).

14 Manel N, Kim FJ, Kinet S, Taylor N, Sitbon M, Battini J-L. The ubiquitous glucose transporter GLUT-1 is a receptor for HTLV. Cell 115(4), 449-459 (2003).

15 Dalod M, Chelbi R, Malissen B, Lawrence T. Dendritic cell maturation: functional specialization through signaling specificity and transcriptional programming. EMBO J. 33(10), 1104-1116 (2014).

16 Cavrois M, Neidleman J, Greene WC. The achilles heel of the trojan horse model of HIV-1 trans-infection. PLoS Pathog. 4(6), e1000051 (2008).

17 Rizkallah G, Alais S, Futsch N et al. Dendritic cell maturation, but not type I interferon exposure, restricts infection by HTLV-1, and viral transmission to T-cells. PLoS Pathog. 13(4), e1006353 (2017).

18 Banchereau R, Baldwin N, Cepika A-M et al. Transcriptional specialization of human dendritic cell subsets in response to microbial vaccines. Nat. Commun. 5, 5283 (2014). 
19 Cachat A, Chevalier SA, Alais S et al. Alpha interferon restricts human T-lymphotropic virus type 1 and 2 de novo infection through PKR activation. J. Virol. 87(24), 13386-13396 (2013).

20 Kinpara S, Hasegawa A, Utsunomiya A et al. Stromal cell-mediated suppression of human T-cell leukemia virus type 1 expression in vitro and in vivo by type I interferon. J. Virol. 83(10), 5101-5108 (2009).

21 Nasr R, Marçais A, Hermine O, Bazarbachi A. Overview of targeted therapies for adult T-cell Leukemia/Lymphoma. Methods Mol. Biol. Clifton NJ 1582, 197-216 (2017).

22 Pique C, Lagaudrière-Gesbert C, Delamarre L, Rosenberg AR, Conjeaud H, Dokhélar MC. Interaction of CD82 tetraspanin proteins with HTLV-1 envelope glycoproteins inhibits cell-to-cell fusion and virus transmission. Virology. 276(2), 455-465 (2000).

23 Manel N, Battini J-L, Sitbon M. Human T cell leukemia virus envelope binding and virus entry are mediated by distinct domains of the glucose transporter GLUT1. J. Biol. Chem. 280(32), 29025-29029 (2005).

24 Marsh M, Helenius A. Virus entry: open sesame. Cell 124(4), 729-740 (2006).

25 Savina A, Jancic C, Hugues S et al. NOX2 controls phagosomal pH to regulate antigen processing during crosspresentation by dendritic cells. Cell. 126(1), 205-218 (2006).

26 Ribeiro CMS, Sarrami-Forooshani R, Setiawan LC et al. Receptor usage dictates HIV-1 restriction by human TRIM5 $\alpha$ in dendritic cell subsets. Nature 540(7633), 448-452 (2016). 